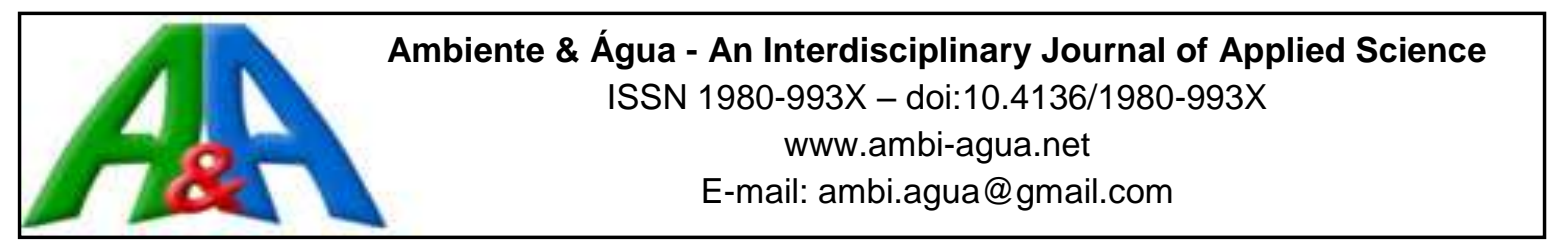

\title{
Qualidade da água superficial por meio de análise do componente principal
}

\author{
doi:10.4136/ambi-agua.1468
}

Received: 23 Jul. 2014; Accepted: 13 Aug. 2015

\section{Nícolas Reinaldo Finkler ${ }^{1 *}$; Denise Peresin ${ }^{1}$; Jardel Cocconi ${ }^{1}$; Taison Anderson Bortolin ; Adivandro Rech²; Vania Elisabete Schneider ${ }^{1}$}

\footnotetext{
${ }^{1}$ Universidade de Caxias do Sul - Instituto de Saneamento Ambiental (ISAM), Caxias do Sul, RS, Brasil

${ }^{2}$ Secretaria Municipal do Meio Ambiente de Caxias do Sul, Caxias do Sul, RS, Brasil

*Autor correspondente: e-mail: nicolas.finkler@gmail.com, deniseperesin@gmail.com, jardelcocconi@gmail.com, taisonbortolin@yahoo.com.br, arech@caxias.rs.gov.br, veschnei@ucs.br
}

\section{RESUMO}

Os fatores naturais e, em especial os antrópicos, que contribuem para a variação espacial e temporal da qualidade da água superficial nas bacias hidrográficas do município de Caxias do Sul foram determinados com uso de técnicas multivariadas de análise de dados. Foi utilizada a técnica de Análise do Componente Principal (ACP) como base para o estudo. $\mathrm{O}$ monitoramento foi realizado em 30 pontos de monitoramento no período compreendido entre setembro de 2012 a janeiro de 2014. A amostragem foi realizada bimestralmente, abrangendo seis bacias hidrográficas, onde foram analisados 21 parâmetros físicos, químicos e biológicos. Os resultados obtidos demonstraram que com o emprego da ACP, foi possível explicar uma variância total de $71,3 \%$ para os dados de qualidade de água. Ainda, constatou-se que os principais fatores que contribuem para a variação da qualidade da água nas bacias hidrográficas da região são a poluição doméstica e industrial, sobretudo do setor galvonotécnico. Foi verificada por fim, uma tendência à atenuação dos poluentes nos corpos hídricos à jusante das áreas urbanas e de grande influência antrópica, especialmente na medida em que há menor pressão das áreas urbanizadas sobre as bacias que drenam a região.

Palavras-chave: monitoramento de recursos hídricos, gestão de recursos hídricos, técnicas estatísticas multivariadas.

\section{Surface water quality data by principal component analysis}

\begin{abstract}
This study used multivariate techniques for data analysis in order to determine the natural and anthropogenic factors that contribute to the spatial and temporal variations of water quality in urban watersheds of Caxias do Sul, Brazil. Principal Component Analysis (PCA) was used to analyze data collected at 30 points between September 2012 and January 2014. Monitoring was conducted bimonthly in six urban basins, where a total of 21 physical, chemical and biological parameters were analyzed. We found that PCA can explain $71.3 \%$ of the total variance in water quality, and that domestic and industrial pollution are the main
\end{abstract}


contributors to the water quality variation in the region, especially from the galvanic manufacturing sector. Furthermore, we observed a trend of self-attenuation of pollutants in water downstream from urban areas and great anthropogenic influence as the pressure from urbanized areas decreases.

Keywords: multivariate statistical techniques, water resources monitoring, water resources management.

\section{INTRODUÇÃO}

As bacias hidrográficas localizadas próximas a áreas urbanas são caracterizadas pela vulnerabilidade ao lançamento de efluentes industriais e domésticos. Os processos naturais de contaminação das bacias hidrográficas incluem a precipitação pluviométrica, carregamento de sedimentos e erosão, além dos processos antropogênicos como industrialização, urbanização e agricultura, com grande contribuição para a degradação dos recursos hídricos e determinantes na qualidade das águas (Singh et al., 2004).

O método usual de avaliação de qualidade da água consiste em medir múltiplos parâmetros de poluentes em diferentes estações de monitoramento, de características topográficas distintas e com frequência periódica. Como resultado, obtém-se uma matriz complexa composta por diversos parâmetros e grandezas de difícil leitura e interpretação. Em razão da dificuldade apresentada, novos métodos de representação de resultados enxutos e concisos com a realidade de uma bacia hidrográfica são buscados (Fan et al., 2010).

Nesse cenário, as técnicas de análise multivariada, como análise de componente principal (ACP), análise de agrupamento (AA), análise discriminante (AD) e análise fatorial (AF), vêm sendo largamente empregadas na interpretação de dados complexos de qualidade de água e de outros problemas ambientais. Essas ferramentas permitem a redução do número de variáveis a um pequeno número de índices (componentes principais ou fatores), de modo a preservar as relações existentes nos dados originais. A aplicação de ACP tem sido uma ferramenta efetiva no gerenciamento de recursos hídricos e controle de poluição, além de ser útil na identificação de possíveis fatores de influência nas bacias hidrográficas (Fan et al., 2010; Ouyang, 2005; Shrestha e Kazama, 2007).

Estudos realizados por Singh et al. (2004), Shrestha e Kazama (2007), Bricker e Jones (1995), Fan et al. (2010), Mustonen et al. (2008), Coletti et al. (2009), Ouyang (2005), Lima et al. (2010) e González et al. (2011) empregaram análise multivariada a dados de qualidade de água em diferentes bacias hidrográficas.

O presente estudo visou analisar e identificar os principais parâmetros de qualidade e as fontes poluidoras relacionadas à variação espacial e temporal da qualidade dos corpos hídricos no município de Caxias do Sul, estado do Rio Grande do Sul - Brasil, pelo uso de técnica multivariada da ACP.

\section{MATERIAIS E MÉTODOS}

\section{1. Área de estudo}

O município de Caxias do Sul localiza-se no Estado do Rio Grande do Sul, na Região Metropolitana da Serra Gaúcha, ocupando uma área de $1.644,3 \mathrm{~km}^{2}$. A população estimada no ano de 2014 foi 470.223 habitantes, dos quais aproximadamente 96\% vivem na área urbana e $4 \%$ na área rural. A densidade demográfica gira em torno de $265 \mathrm{hab} / \mathrm{km}^{2}$. Caracteriza-se por uma grande gama de atividades econômicas, dentre elas indústrias metal-mecânicas, têxteis, e transformação de plásticos, além de hortifrutigranjeiros, suinocultura, avicultura e pecuária (IBGE, 2014). Detém o segundo maior Produto Interno Bruto do estado, atrás somente da capital Porto Alegre (FEE, 2012). 
O município está localizado sobre o divisor das bacias hidrográficas Taquari-Antas e Caí, contribuintes da região hidrográfica do Guaíba. A região norte do município insere-se na bacia hidrográfica do rio Taquari-Antas e contribui com $65 \%$ do território municipal. A cidade é drenada pelas microbacias dos rios Tega e São Marcos, as quais possuem relevantes porções localizadas na área urbana. A região sul do município está inserida na bacia hidrográfica do rio Caí e representa $35 \%$ do território municipal. Neste sistema destacam-se as microbacias dos arroios Belo, Pinhal e do Ouro, e do rio Piaí.

Desta forma, considerando que o município possui significativa extensão e distintas áreas de elevada urbanização, uma área rural voltada essencialmente à olericultura e fruticultura, além do fato de que a área urbana situa-se em uma região de nascentes, Caxias do Sul, torna-se uma área de relevante interesse na avaliação da qualidade da água e do impacto ocasionado pelos diferentes usos dos solos existentes na região.

Os dados de qualidade de água das seis bacias hidrográficas analisadas são referentes ao período de setembro de 2012 a janeiro de 2014, com periodicidade bimestral de coleta. Para alocação dos pontos de coleta foram considerados como critérios uso e ocupação do solo e área de drenagem de cada curso d'água, buscando assim maior representatividade das bacias. A localização dos pontos que compõem a rede de amostragem é apresentada na Figura 1, bem como a localização em relação ao município e à bacia hidrográfica pertencente. Por sua vez, a Tabela 1 apresenta a descrição de cada ponto de monitoramento.

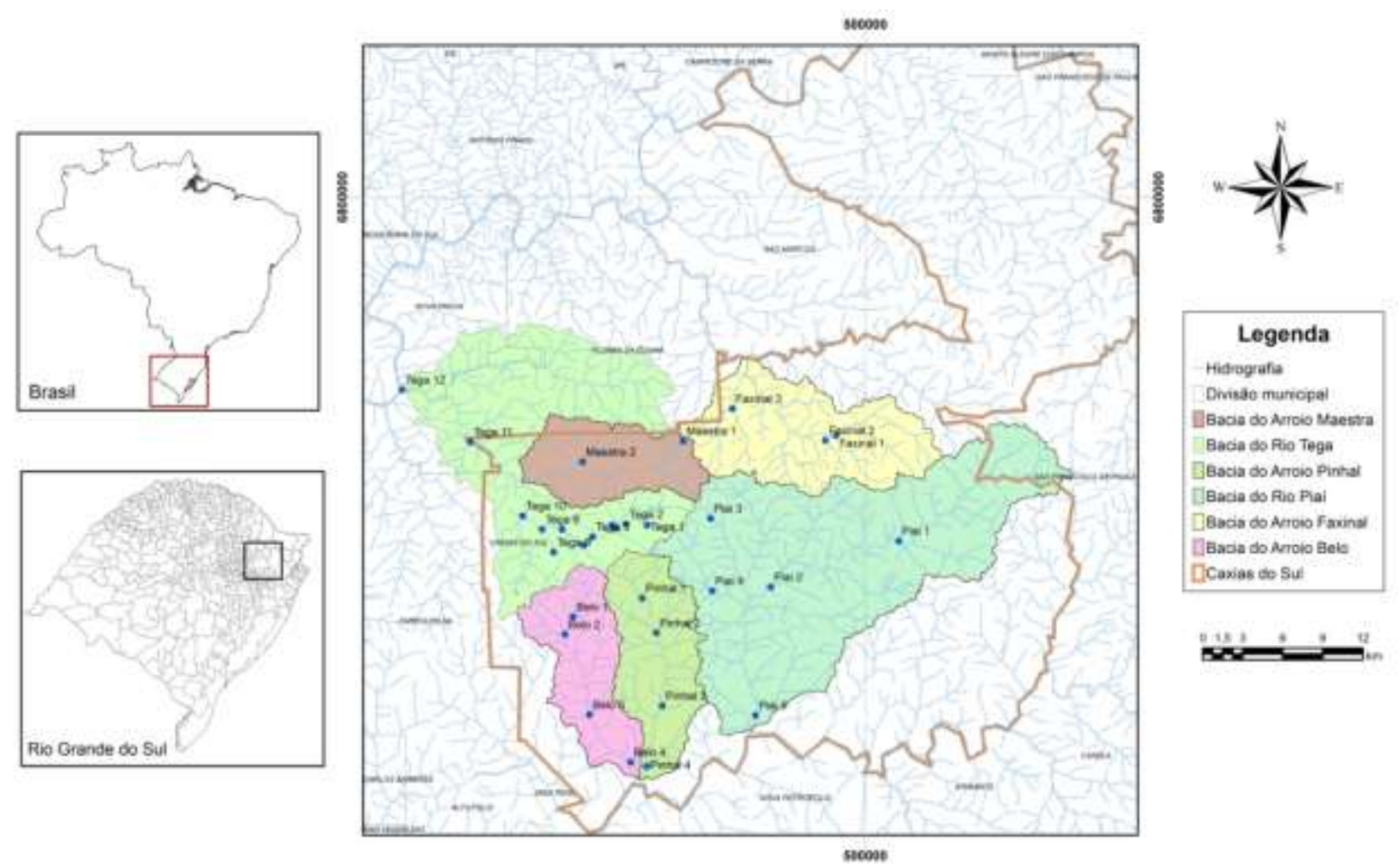

Figura 1. Contexualização dos pontos de monitoramento e das bacias hidrográficas analisadas em relação à divisão municipal e hidrografia.

\subsection{Análise dos dados - Análise de Componentes Principais (ACP)}

A ACP é definida como uma técnica estatística multivariada que transforma o conjunto de dados em um novo sistema de variáveis. Nesse novo arranjo, os componentes principais (CP) são funções lineares do conjunto original de dados, aos quais, não possuem correlação. A projeção da maior variação da amostra gera o primeiro $\mathrm{CP}$, a projeção da segunda maior variação, o segundo CP, e assim por diante (Jolliffe, 2002). 
Tabela 1. Características dos pontos monitorados.

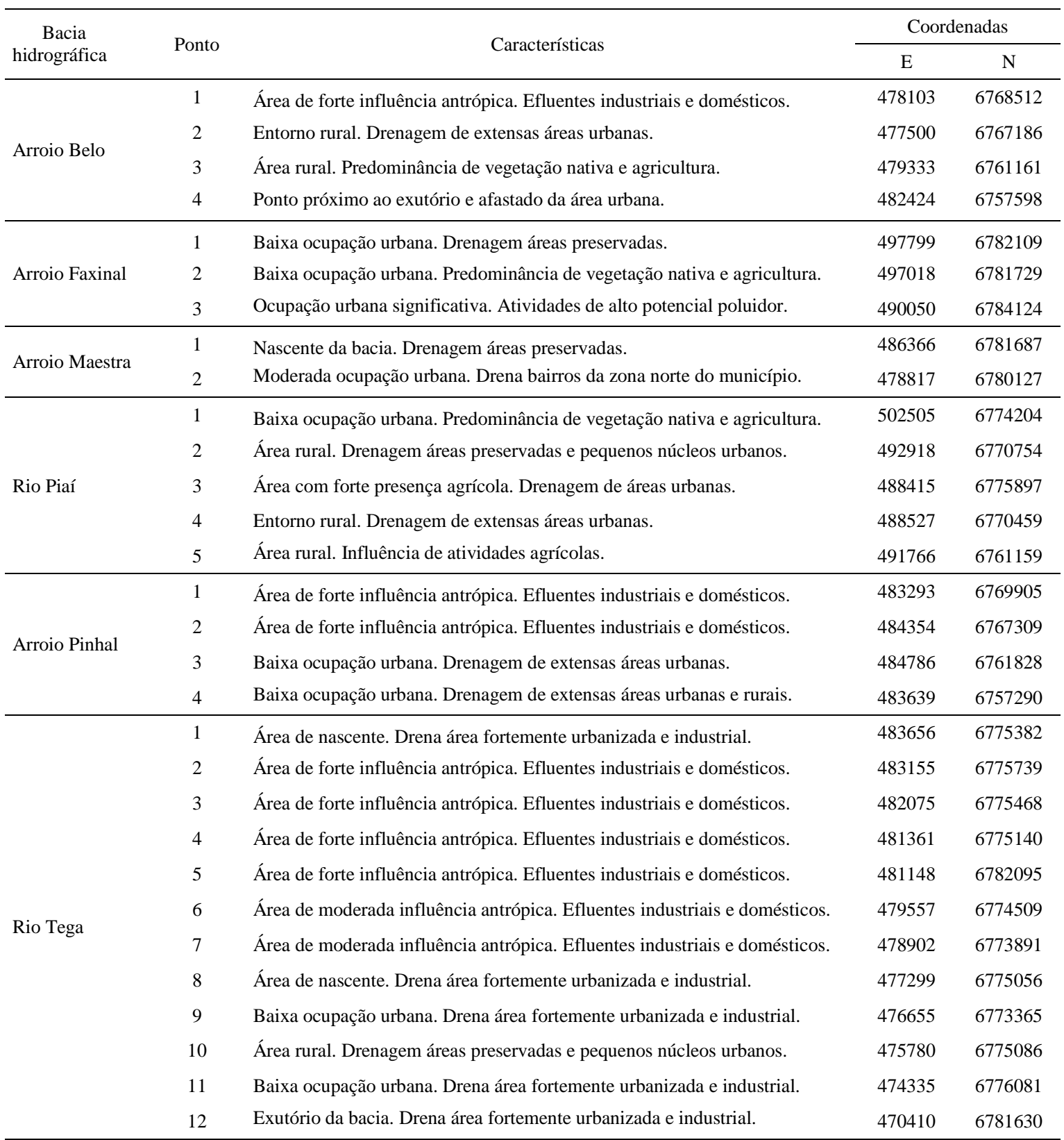

Matematicamente, a ACP envolve cinco passos principais: (i) a padronização dos valores utilizando média zero e variância 1, para assegurar que os mesmos possuirão pesos iguais na análise, conforme Equação 1; (ii) cálculo da matriz de correlações R ou matriz de co-variância $\Sigma$; (iii) determinação dos autovetores $\lambda 1, \lambda 2, \ldots, \lambda \mathrm{p}$ e seus correspondentes autovalores a1, a2,...,ap pela Equação 2; (iv) descarte de componentes que fazem parte de uma pequena proporção na variância de dados; e (v) desenvolvimento da matriz de cargas fatoriais com aplicação ou não de métodos de rotação de variáveis à matriz, visando à diminuição do número de CP (Ouyang, 2005).

$$
\begin{aligned}
& Z_{i j}=\frac{x_{i j}-\bar{x}_{j}}{s\left(x_{j}\right)}, \text { sendo } i=1,2, \ldots, \text { ne } j=1,2, \ldots, p \\
& |R-I \lambda| \text { ou } \operatorname{det}[R-I \lambda]=0
\end{aligned}
$$


em que:

$x_{i v}$ é a característica do elemento $i$;

$\overline{x_{v}}$, o valor médio do elemento $v$;

$\lambda$, os auto vetores; $v$, níveis de liberdade; $\mathrm{e}$

$|R|$, o determinante da matriz de correlações.

O critério de seleção dos CP consiste em incluir somente os componentes cujos autovalores próprios sejam superiores a 1. O critério sugerido por Kaiser (1958), também tende a incluir poucos componentes quando o número de parâmetros originais é inferior a vinte. Em geral, utilizam-se os componentes que conseguirem sintetizar uma variância acumulada em torno de $70 \%$ da variância total dos dados (Mardia et al., 1979). Liu et al. (2000) classifica os valores das cargas fatoriais, correspondentes à composição absoluta dos $\mathrm{CP}$, como relevante $(>0,75)$, médio $(>0,50$ e $<0,75)$ e irrelevante $(<0,50)$. Nesse estudo, como se trata de dados de qualidade de água, os quais apresentam grande variabilidade, consideraram-se na composição de cada $\mathrm{CP}$, variáveis cujas cargas fatoriais sejam superiores a 0,50 .

No estudo realizado, ainda procedeu-se a rotação das variáveis para que os resultados fossem apresentados com maior coesão. Para tal foi escolhido o método de rotação VARIMAX (Kaiser, 1958). Trata-se de um método bastante utilizado em pesquisas estatísticas multivariadas, uma vez que torna a interpretação mais eficiente e simplificada ao reduzir o número de correlações entre as variáveis (Abdi, 2003).

A aplicação da ACP depende de dois testes que devem ser realizados previamente, confirmando a adequação da técnica aos dados de que se dispõe: teste de Kaiser-Meyer-Olkin (KMO) e teste de Bartlett.

O teste de KMO verifica a medida de correlação da matriz como um todo, isto é, a correlação entre as variáveis independentes. Ferreira Jr. et al. (2004) citam que o teste KMO é um identificador que compara a magnitude do coeficiente de correlação observado com a magnitude do coeficiente de correlação parcial. Os valores do teste de KMO variam de 0 a 1 . Considera-se que valores de KMO abaixo de 0,5 indicam que os dados não são adequados para aplicação da ACP. Alguns autores, como Rencher (2002), sugerem que, para que um modelo de análise de componentes principais possa ser adequadamente ajustado aos dados, é necessário que a matriz de correlação inversa $R_{p x p}{ }^{-1}$ seja próxima da matriz diagonal. A medida de adequabilidade da amostra KMO é representada pelo índice MAS, calculado pela Equação 3.

$$
M A S=\frac{\sum j \neq k \sum j \neq k r_{j k}^{2}}{\sum j \neq k \sum j \neq k r_{j k}^{2}+\sum j \neq k \sum j \neq k q_{j k}^{2}}
$$

em que:

$r_{j k}^{2}$ é o quadrado dos elementos da matriz de correlação original (fora da diagonal);

$q_{j k}^{2}$ é o quadrado dos elementos fora da diagonal da matriz anti-imagem (onde $q_{j k}$ é o coeficiente de correlação parcial entre as variáveis $X j$ e $X k$ ).

O teste de esfericidade de Bartlett testa se a matriz de correlação é uma matriz identidade, o que indicaria que não há correlação entre os dados. Dessa forma, procura-se, para um nível de significância assumido em 5\%, rejeitar a hipótese nula de matriz de correlação identidade. A hipótese básica diz que a matriz de correlação da população é uma matriz identidade, a qual indica que o modelo fatorial é inapropriado. A estatística do teste é dada a partir da Equação 4. 


$$
x^{2}=-\left[(n-1)-\frac{2 p+5}{6}\right] \times \ln |R|
$$

A estatística tem distribuição qui-quadrado $\left(x^{2}\right)$, com grau de liberdade $(v)$ dado pela Equação 5.

$$
v=\frac{p(p-1)}{2}
$$

em que:

$n$ expressa o tamanho da amostra;

$p$, o número de variáveis; e

$|R|$, o determinante da matriz de correlação.

\section{RESULTADOS E DISCUSSÃO}

A apresentação dos resultados da aplicação da ACP desse estudo é dividida em duas partes. A primeira expõe uma análise conjunta das seis bacias do município de Caxias do Sul, com o objetivo de visualizar o arranjo dos parâmetros que influenciam a variação da qualidade da água de maneira global. A segunda parte, cujo resultado é ordenado em função dos componentes obtidos, apresenta uma análise individual por bacia hidrográfica. Essa análise busca ordenar parâmetros e pontos de amostragem, e tem como objetivo identificar os processos que exercem maior pressão dos dados de qualidade de água.

\subsection{Análise conjunta das seis bacias hidrográficas}

$\mathrm{O}$ teste KMO apresentou índice igual a 0,850 e o teste de Bartlett foi altamente significativo $\left(\mathrm{x}^{2}(120)=3195,61\right.$ e p-valor < 0,001); portanto, a aplicação da ACP é apropriada a presente amostra. A ACP realizada conjuntamente entre todas as bacias revelou a existência de cinco $\mathrm{CP}$, sendo que a variância explicada foi de $71,3 \%$ para os dados de qualidade da água. Na Tabela 2, apresentam-se a pontuação dos autovalores de cada $\mathrm{CP}$, bem como a distribuição das cargas fatoriais e correlações dos mesmos com as variáveis rotacionadas.

De acordo com os resultados apresentados na Tabela 2, o CP1 explicou uma variação de $27,1 \%$, e está formado pelos parâmetros $\mathrm{NH}_{3}-\mathrm{N}$, NTK, Coliformes termotolerantes, DBO, DQO, Sólidos Totais e Oxigênio dissolvido, esse representado por um coeficiente negativo. A associação desses parâmetros indica que as águas do município sofrem contaminação por meio de despejos de efluentes domésticos in natura ou parcialmente tratados nos corpos hídricos. Considera-se esperada tal condição, uma vez à época do monitoramento, as estações de tratamento de esgotos (ETE) do Tega, Pinhal e Belo não estavam em sua operação total ou encontravam-se em fase final de construção.

O CP2 correspondeu a $20,2 \%$ da variação total dos dados de qualidade de água e sugere que a contaminação química da água, seja por despejos de efluentes industriais, bem como a utilização de agroquímicos na área rural, é relevante na variação da qualidade da água nas bacias analisadas. $\mathrm{O}$ resultado é explicado pelo pujante centro industrial que o município de Caxias do Sul possui, conglomerando diversas atividades industriais, principalmente do ramo galvanotécnico, cujos padrões de lançamento de efluentes não alcançam o disposto em dispositivos legais de controle de poluição. $\mathrm{O} \mathrm{CP}$ pode sugerir que processos naturais também inferem na determinação da qualidade de água, tendo em vista a presença de Alumínio na composição geológica da região de estudo. Ainda, constatou-se, que os parâmetros Sólidos totais, DQO e DBO obtiveram cargas significativas, tanto no CP1, quanto no CP2. Tais resultados indicam que o transporte de sedimentos contaminados tanto por poluição doméstica, industrial ou rural, pode interferir na qualidade da água. 
Tabela 2. Cargas fatoriais com rotação das variáveis para os $\mathrm{CP}$ encontrados.

\begin{tabular}{|c|c|c|c|c|c|}
\hline \multirow{2}{*}{ Parâmetros } & \multicolumn{5}{|c|}{ Componente principal } \\
\hline & 1 & 2 & 3 & 4 & 5 \\
\hline Nitrogênio Amoniacal & $\mathbf{0 , 8 1 7}$ & 0,206 & 0,370 & $-0,129$ & 0,079 \\
\hline Nitrogênio Total Kjeldahl & 0,803 & 0,387 & 0,321 & $-0,104$ & 0,046 \\
\hline Oxgênio Dissolvido & $-0,758$ & $-0,060$ & $-0,116$ & 0,362 & $-0,119$ \\
\hline DBO & 0,754 & 0,551 & $-0,007$ & 0,014 & $-0,028$ \\
\hline Coliformes Termotolerantes & $\mathbf{0 , 7 3 9}$ & 0,086 & $-0,158$ & 0,057 & $-0,067$ \\
\hline Sólidos Totais & 0,645 & 0,588 & 0,201 & $-0,153$ & 0,073 \\
\hline DQO & 0,662 & 0,687 & 0,011 & $-0,006$ & 0,006 \\
\hline Alumínio Total & 0,009 & 0,860 & $-0,234$ & 0,064 & 0,029 \\
\hline Condutividade & 0,422 & 0,755 & 0,195 & $-0,134$ & 0,085 \\
\hline Zinco Total & 0,160 & 0,752 & 0,301 & $-0,022$ & $-0,083$ \\
\hline Cianeto & 0,013 & $-0,002$ & 0,754 & $-0,111$ & $-0,080$ \\
\hline $\mathrm{pH}$ & 0,143 & 0,111 & 0,689 & 0,256 & 0,098 \\
\hline Temperatura da água & 0,229 & 0,004 & $-0,010$ & $-0,800$ & $-0,012$ \\
\hline Fósforo Total & 0,112 & $-0,045$ & $-0,061$ & 0,106 & 0,905 \\
\hline Fenol & 0,430 & $-0,168$ & $-0,195$ & 0,226 & $-0,406$ \\
\hline Chumbo Total & 0,015 & $-0,021$ & 0,030 & 0,464 & 0,023 \\
\hline Total & 4,330 & 3,244 & 1,588 & 1,203 & 1,049 \\
\hline \% Variação & 27,061 & 20,272 & 9,925 & 7,519 & 6,558 \\
\hline \% Variação Acumulada & 27,061 & 47,334 & 57,259 & 64,777 & 71,335 \\
\hline
\end{tabular}

Nota: Método de rotação de variáveis: VARIMAX com normalização de Kaiser.

De forma geral, os demais $\mathrm{CP}$ são resultados da pressão que atividades antropogênicas exercem sobre os corpos hídricos. O CP3 explicou uma variação de 9,9\% e os parâmetros que o compõem ( $\mathrm{pH}$ e Cianeto) representam a contaminação química da água. O CP4 apresentou uma correlação negativa com a temperatura da água, correspondendo a uma variação de 7,5\% nos dados. O parâmetro Fósforo Total isoladamente representou o CP5, explicou uma variação de $6,5 \%$ da qualidade da água e apresenta a maior carga fatorial entre todos os parâmetros em todos os $\mathrm{CP}(0,905)$. A presença de grandes concentrações desse parâmetro em corpos hídricos ocasiona processos de eutrofização e está associada à contaminação doméstica, industrial e agrícola.

\subsection{Análise individual das Bacias Hidrográficas}

Na Figura 2, apresentam-se os gráficos que representam a ordenação da ACP para cada bacia hidrográfica analisada.

De forma geral, os resultados apresentados em cada bacia hidrográfica mostraram-se similares aos obtidos na ACP anterior. Os parâmetros indicativos de poluição doméstica tais como DBO, P, DQO, $\mathrm{NH}_{3}-\mathrm{N}$, NTK e coliformes termotolerantes contribuíram para a ordenação do primeiro eixo, explicando a maior variação da qualidade da água em cada bacia. $\mathrm{Na}$ presente $\mathrm{ACP}$, tais parâmetros alcançaram valores de $\mathrm{r}>0,7$, e em bacias mais urbanizadas, $r>0,9$. Como já citado, esses resultados eram esperados, tendo em vista a não adequação do gerenciamento de esgotos domésticos nas bacias do município, sendo situação comum, o lançamento de forma direta nos corpos hídricos. 
Nesse eixo é possível observar que o parâmetro Oxigênio Dissolvido está ordenado no lado negativo do eixo, indicando que, ainda que os corpos hídricos sofram impacto devido a atividades antrópicas. O processo de atenuação dos poluentes é recorrente em todas as bacias hidrográficas analisadas, muito devido à diminuição da pressão antrópica no sentido jusante, como à reoxigenação dos rios, propiciada pelo relevo local.

$\mathrm{O}$ segundo eixo foi ordenado em função de parâmetros como $\mathrm{pH}$, condutividade e sólidos totais $(0,6<\mathrm{r}<0,8)$ em todas as bacias, com exceção da bacia do Arroio Faxinal, ordenada em função do parâmetro fenol $(r>0,9)$. A situação observada nessa bacia pode indicar potencial poluição oriunda de indústrias de processamento da borracha, colas e adesivos, resinas impregnantes, componentes elétricos (plásticos) e ou siderúrgicas (CETESB, 2014), as quais estão localizadas dentro da área de drenagem dos pontos amostrados. Nas demais bacias, a ordenação do segundo eixo pelos parâmetros já citados pode indicar a contaminação através de componentes químicos utilizados em outros processos industriais ou aplicados em área rural, corroborando com os resultados da ACP anterior.
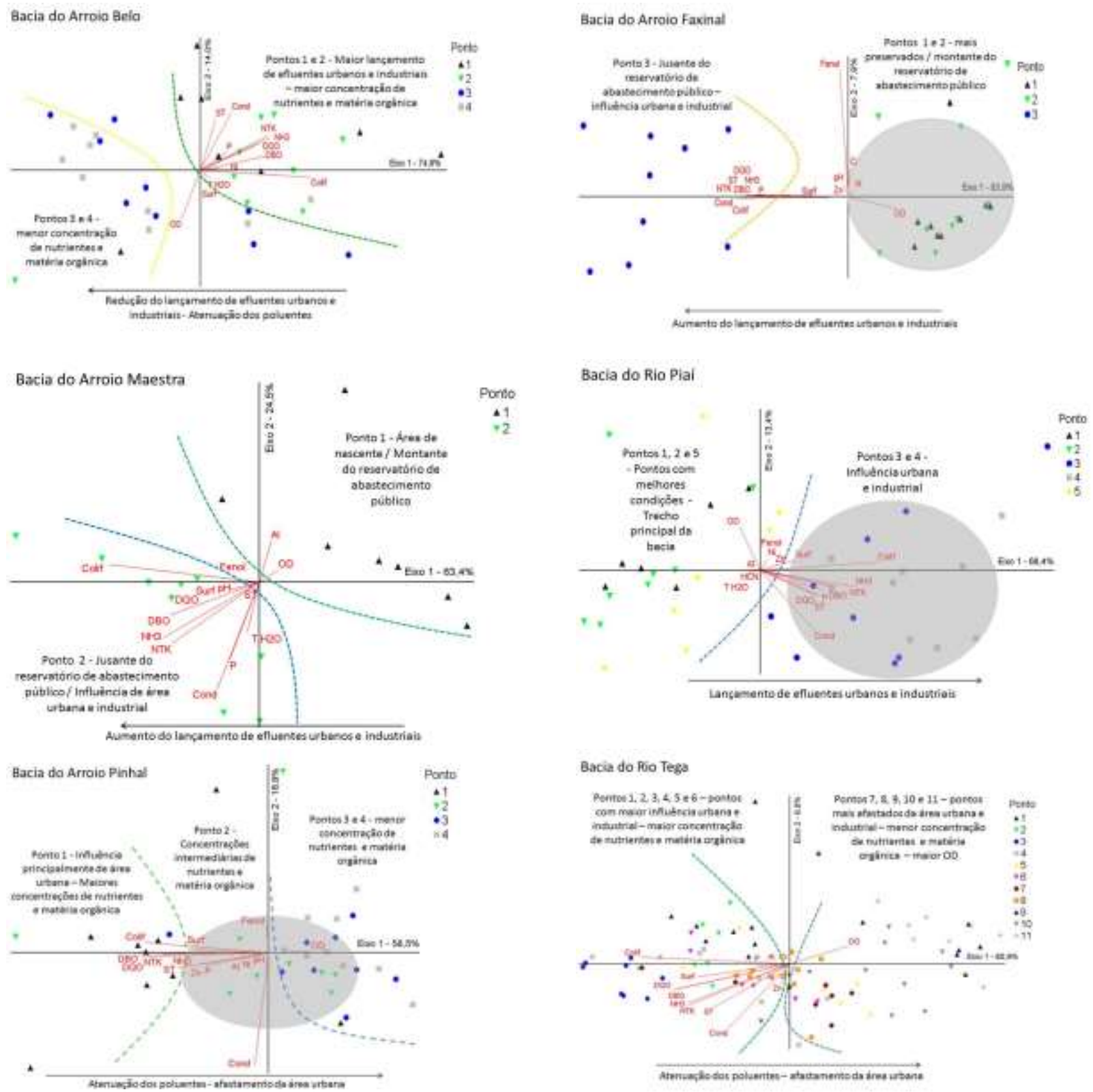

Figura 2. Ordenação da ACP para as bacias hidrográficas do município de Caxias do Sul. 
Os pontos de amostragem, que sofrem maior pressão de atividades antrópicas foram ordenados no lado associado a grandes valores de DBO, P, DQO, $\mathrm{NH}_{3}-\mathrm{N}, \mathrm{NTK}$ e coliformes termotolerantes do eixo 1. O comportamento se deu, provavelmente, ao lançamento de efluentes domésticos e industriais, visto a proximidade destas áreas a zonas urbanizadas.

Por outro lado, os pontos localizados em áreas de menor pressão antrópica ficaram ordenados no lado oposto do eixo 1, no qual encontram-se as menores concentrações de poluentes e maior presença de oxigênio dissolvido, corroborando com o já comentado anteriormente, sobre o potencial de atenuação dos poluentes no recurso hídrico. Estes pontos estão localizados à jusante dos anteriores e a maior distância da área urbana. Destacam-se aqui também os pontos localizados a montante de reservatórios de água para abastecimento público, no caso o Maestra 1 e Faxinal 1. Apesar de estarem localizadas em área de preservação em ambos os pontos verifica-se a presença de metais, como o Alumínio e o Cromo.

\section{CONCLUSÕES}

Por meio da ACP verificou-se que os parâmetros mais significativos na variação da qualidade da água nas bacias hidrográficas de Caxias do Sul estão relacionados às atividades de caráter antrópico. Parâmetros indicadores de matéria orgânica e nutrientes compõem o componente que explicou a porcentagem majoritária $(27,1 \%)$ de variância dos dados de qualidade. Lançamentos de efluentes industriais sem tratamento adequado explicaram 20,3\% da variação dos dados de qualidade da água no município. Outros fatores que refletem a contaminação química da água, como cianetos e pH, sugerem uma variação de 9,9\% e são representados pelo $\mathrm{CP} 3$.

A análise destes componentes indica que o declínio da qualidade da água no município decorre quase que exclusivamente devido à falta de tratamento de efluentes domésticos e industriais, bem como o efeito que cargas difusas sobre os corpos da água. Além disso, podem ser citadas, as atividades relativas ao meio rural, fato evidenciado pela presença de parâmetros relativos à carga orgânica em áreas de menor concentração urbana.

Com relação à ACP realizada de forma individual nas bacias hidrográficas, observa-se um comportamento comum no que se refere à qualidade da água próxima aos centros urbanizados e industriais, agrupando estes pontos principalmente em função dos parâmetros: coliformes termotolerantes, DBO, DQO, $\mathrm{P}, \mathrm{NH}_{3}-\mathrm{N}$ e NTK. Nos cursos d'água com grande extensão, como por exemplo, o Arroio Belo, Arroio Pinhal e Rio Tega, observa-se um potencial de atenuação dos recursos hídricos, favorecido em parte pela geografia local. Contudo, a descarga dos efluentes domésticos e industriais além dos limites de capacidade de atenuação de poluentes nos corpos hídricos locais, impossibilita o processo de depuração de forma efetiva.

Embora o município de Caxias do Sul, RS esteja investindo na implantação de estações de tratamento de efluentes, a rede coletora ainda é pequena para o esgoto gerado, precisando ser ampliada. Portanto, por enquanto, o lançamento de efluentes in natura ou sem o devido tratamento, ainda contribui significativamente para a redução da qualidade da água dos recursos hídricos estudados.

\section{AGRADECIMENTOS}

Os autores gostariam de agradecer a Prefeitura de Caxias do Sul-RS pelo financiamento do projeto. 


\section{REFERÊNCIAS}

ABDI, H. Factor rotations in factor analyses. In: LEWIS-BECK, M.; BRYMAN, A.; FUTING, T. (Eds.). Encyclopedia of Social Sciences. Research Methods. Thousand Oaks: The University of Texas at Dallas, 2003. p.1-8.

BRICKER, O. P.; JONES, B. Main factors affecting the composition of natural waters. In: SALBU, B.; STEINNES, E. Trace elements in natural waters. Boca Raton: CRC Press, 1995. p. 1-5.

COLETTI, C.; TESTEZLAFL, R.; RIBEIRO, T. A. P.; SOUZA, R. T. G. de; PEREIRA, D. de A. Water quality index using multivariate factorial analysis. Revista Brasileira de Engenharia Agrícola e Ambiental, Campina Grande, v. 14, n. 5, p. 517-522, 2009. http://dx.doi.org/10.1590/S1415-43662010000500009

COMPANHIA AMBIENTAL DO ESTADO DE SÃO PAULO - CETESB. Variáveis de qualidade das águas. 2014. Disponível em: < http://aguasinteriores.cetesb.sp.gov.br/ publicacoes-e-relatorios/>. Acesso em: 23 jun. 2015.

FAN, X.; CUI, B.; ZHAO, H.; ZHANG, Z.; ZHANG, H. Assessment of river water quality in Pearl River Delta using multivariate statistical techniques. Procedia Environmental Sciences, v. 2, p.1220-1234, 2010.

http://dx.doi.org/10.1016/j.proenv.2010.10.133

FUNDAÇÃO DE ECONOMIA E ESTATÍSTICA - FEE. Indicadores: PIB municipal. 2012. Disponível em: <http://www.fee.rs.gov.br/indicadores/pib-rs/municipal/tabelasdestaques/>. Acesso em: 03 ago. 2015.

FERREIRA JR., S.; BAPTISTA, A. J. M. S.; LIMA, J. E. de. A modernização agropecuária nas microrregiões do estado de Minas Gerais. RER, Rio de Janeiro, v. 42, n. 01, p. 7389, 2004.

GONZÁlEZ, O.; ALMEIDA, C.; QUINTAR, S.; MALLEA, M.; GONZÁlEZ, P. Application of multivariate statistical techniques to evaluate organic pollution on a river in Argentina. Rev. Ambient. Água, Taubaté, v. 6, n. 3, p. 27-42, 2011. http://dx.doi.org/10.4136/ambi-agua.696

INSTITUTO BRASILEIRO DE GEOGRAFIA E ESTATÍSTICA - IBGE. Produto Interno Bruto dos municípios 2014. Disponível em: <http://cidades.ibge.gov.br/comparamun/ compara.php? coduf $=43 \&$ idtema $=103 \& \operatorname{codv}=$ v05\&order $=$ dado $\&$ dir $=$ desc $\&$ lista $=$ uf $\&$ cus tom=>. Acesso em: 6 Sep. 2014.

JOLLIFFE, I. T. Principal component analysis. $2^{\text {th }}$ ed. New York: Springer-Verlag New York, 2002. 487 p.

KAISER, H. F. The varimax criterion for analytic rotation in factor analysis. Psychometrika, v. 23, n. 03, p. 187-200, 1958. http://dx.doi.org/10.1007/BF02289233

LIMA, F.; AMANAJÁS, J.; GUEDES, R.; SILVA, E. Análises de Componente Principal e de Agrupamento para estudo de ventos para a geração de energia eólica na região do Ceará, Paraíba, Pernambuco e Rio Grande do Norte, Brasil. Rev. Ambient. Água, Taubaté, v. 5, p. 188-201, 2010. http://dx.doi.org/10.4136/ambi-agua.147 
LIU S.; MANSON, J. E.; STAMPFER, M. J.; HU, F. B.; GIOVANNUCCI, E.; COLDITZ, G. A. et al. A prospective study of whole-grain intake and risk of type 2 diabetes mellitus in US women. American Journal of Public Health, v. 90, n. 9, p. 1409-1415, 2000.

MARDIA, K. V.; KENT, J. T.; BIBBY, J. Multivariate analysis. London: Academic, 1979.

MUSTONEN, S. M.; TISSARI, S.; HUIKKO, L.; KOLEHMAINEN, M.; LEHTOLAB, M. J.; HIRVONEN, A. Evaluating online data of water quality changes in a pilot drinking water distribution system with multivariate data exploration methods. Water Research, Amsterdam, v. 42, p. 2421-2430, 2008. http://dx.doi.org/10.1016/j.watres.2008.01.015

OUYANG, Y. Evaluation of river water quality monitoring stations by principal component analysis. Water Research, Amsterdam, v. 39, n. 12, p. 2621-2635, 2005. http://dx.doi.org/10.1016/j.watres.2005.04.024

RENCHER, A. Methods of multivariate analysis. New York: John Wiley \& Son, 2002. 738 p.

SHRESTHA, S; KAZAMA, F. Assessment of surface water quality using multivariate statistical techniques: a case study of the Fuji river basin, Japan. Environmental Modelling \& Software, v. 22, n. 4, p. 464-475, 2007. http://dx.doi.org/10.1016/j.envsoft.2006.02.001

SINGH, K. P.; MALIK, A.; MOHAN, D.; SINHA, S. Multivariate statistical techniques for the evaluation of spatial and temporal variations in water quality of Gomti River (India) - a case study. Water Research, Amsterdam, v. 38, n. 18, p. 3980-3992, 2004. http://dx.doi.org/10.1016/j.watres.2004.06.011 